\section{Symmetries of bilingual language switch costs in conflicting versus non-conflicting contexts*}

\author{
CONG LIU \\ Guangdong Provincial Key Laboratory of Mental Health and \\ Cognitive Science, and Center for Studies of Psychological \\ Application, School of Psychology, South China Normal \\ University, China \\ L U J I AO \\ Beijing Key Laboratory of Applied Experimental Psychology, \\ School of Psychology, Beijing Normal University, China \\ Z IY I WANG \\ Guangdong Provincial Key Laboratory of Mental Health and \\ Cognitive Science, and Center for Studies of Psychological \\ Application, School of Psychology, South China Normal \\ University, China \\ MENGXING WANG \\ Guangdong Provincial Key Laboratory of Mental Health and \\ Cognitive Science, and Center for Studies of Psychological \\ Application, School of Psychology, South China Normal \\ University, China \\ RUIMING WANG \\ Guangdong Provincial Key Laboratory of Mental Health and \\ Cognitive Science, and Center for Studies of Psychological \\ Application, School of Psychology, South China Normal \\ University, China \\ YAN JING W U \\ Faculty of Foreign Languages, Ningbo University, China
}

(Received: May 23, 2017; final revision received: March 14, 2018; accepted: March 15, 2018; first published online 13 June 2018)

Previous studies have demonstrated that language switching in bilinguals can be affected by a number of variables, including the processing context. Here, we used a modified language-switching task combined with a Stroop paradigm, which manipulated the context of the task, to examine the impact of processing context on switch costs. The results of both Experiment 1 and Experiment 2 showed that the switch costs and the level of asymmetry in the switch costs are larger in the conflicting context than in the non-conflicting context, suggesting that the processing context affects the switch costs. In addition, the results of Experiment 2 revealed that individual variances in cognitive control capacity also play a role in the overall magnitude of the switch costs. Critically, processing context effects can be modulated by individual variance in cognitive control capacities. The results of this study are discussed within the framework of classic models of bilingual language control (e.g., the inhibitory control model).

Keywords: switch costs, language switching, conflicting context, language production

\section{Introduction}

Previous studies have shown that speech production in bilinguals involves activating both the target and the non-target language (Costa, Santesteban \& Caño,

\footnotetext{
* This work was supported by the Foundation for innovation team in Guangdong Higher Education (2015WCXTD003 \& 2016WCXTD006), Guangdong Province Universities and colleges Pearl River Younger Scholar Funded Scheme (2016), and the Innovation Project of Graduate School of South China Normal University (2016wkxm13). Yan Jing Wu is sponsored by K. C. Wong Magna Fund in Ningbo University. Cong Liu and Yan Jing Wu contributed equally to this work.
}

2005; Rodriguez-Fornells, Van Der Lugt, Rotte, Britti, Heinze \& Münte, 2005). Therefore, bilinguals need a mechanism to control the potential cross-language interference. According to the inhibitory control model (i.e., ICM, see Green, 1998; Meuter \& Allport, 1999), bilingual speakers control their languages by inhibiting the activation level of the non-target language while accessing lexical representations in the target language. The language-switching paradigm is a commonly used experimental procedure for examining the underlying cognitive processes of bilingual language control (Meuter \& Allport, 1999; Gollan, Kleinman \& Wierenga, 2014; Chang, Xie, Li, Wang \& Liu, 2016). In the classic

Address for correspondence:

Ruiming Wang, PhD, School of Psychology, South China Normal University, 510631 Guangzhou, P. R. China.

wangrm@scnu.edu.cn 
language-switching task, the response languages of two subsequent trials are manipulated between repeated (i.e., same language) and switch (i.e., different languages) conditions. It is typically found that switching from one language to another yields longer reaction times (i.e., the switch cost) than repeating the same language in two subsequent trials (e.g., Costa \& Santesteban, 2004; Declerck \& Philipp, 2015a; Declerck, Philipp \& Koch, 2013).

The magnitude of the switch costs has been established as a psychological index that is positively correlated with the level of difficulty of language control in bilinguals (Hernandez, Dapretto, Mazziotta \& Bookheimer, 2001; Verhoef, Roelofs \& Chwilla, 2010; Guo, Liu, Chen \& $\mathrm{Li}, 2013)$. For example, it has been found that the switch cost is asymmetrical (i.e., L2-L1 switch costs is larger than L1-L2 switch costs) in non-proficient bilinguals (Verhoef, Roelofs \& Chwilla, 2009; Verhoef et al., 2010) and more balanced in proficient bilinguals (Costa \& Santesteban, 2004; Costa, Santesteban \& Ivanova, 2006; Linck, Schwieter \& Sunderman, 2012). According to the ICM, asymmetrical switch costs suggest that nonproficient bilinguals suppress the more dominant L1 during L2 processing to a greater extent than vice versa. Consequently, switching into L1 from L2 involves more difficulty needed to overcome the inhibition of the L1 activation level on the previous trial than switching into L2 from L1 (de Bruin, Roelofs, Dijkstra \& FitzPatrick, 2014; Verhoef et al., 2009; Verhoef et al., 2010). Therefore, nonproficient bilinguals would experience a larger switch cost asymmetry than proficient bilinguals, given the relative dominance of L1 and L2 between the two populations (Costa \& Santesteban, 2004; Wang, Xue, Chen, Xue \& Dong, 2007; Kroll, Bobb, Misra \& Guo, 2008). However, some studies have not replicated asymmetrical effects in the switching task with bilingual participants who have different levels of proficiency between the dominant language and the non-dominant language. For example, Experiment 4 in Costa et al. (2004) found that SpanishCatalan-English trilingual speakers showed symmetrical switch costs when asked to perform a switching task in their L1 and in their much weaker L3, and this pattern of results cannot be accounted for by the ICM.

To account for the discrepancy in previous studies, Liu et al. (Liu, Liang, Zhang, Lu \& Chen, 2015; Liu, Liang, Dunlap, Fan \& Chen, 2016) argued that the language switch cost is not only affected by the proficiency levels of bilinguals' L1 and L2; variances in the domain-general cognitive control ability between bilingual participants could also account for their performance in language-switching tasks. Their studies showed that for non-proficient bilinguals who have different levels of proficiency in L1 and L2, those with high inhibitory control (i.e., the high-IC group) showed symmetrical switch costs and those with low inhibitory control (i.e., the low-IC group) showed asymmetrical switch costs. Additionally, domain-general cognitive control skills training cancelled the asymmetries in the switch costs for low-IC bilinguals but did not affect the pattern of performance for high-IC bilinguals who showed symmetrical switch costs prior to the training (Liu et al., 2016). Moreover, other studies have shown an overlap in the mechanisms between language control and domaingeneral control (e.g., Declerck, Grainger, Koch \& Philipp, 2017; Prior \& Gollan, 2013). These findings suggest that variances in inhibitory control ability between individuals play an important role in modulating language switch costs and that they should be considered within the ICM framework (Liu et al., 2014; Liu et al., 2015; Liu et al., 2016).

In addition to the participant's background variables (e.g., language proficiency and the individual's cognitive control ability), task-dependent factors have also been shown to affect bilingual switch costs (for a review, see Bobb \& Wodniecka, 2013). For example, Declerck \& Philipp (2015b) showed that sentential contexts modulated the magnitude of switch costs in bilinguals; German-English bilinguals learned three types of sentences combinations (with each type containing one German sentence and one English sentence). Then, they produced words based on a memory-based alternating language sequence (L1-L1-L2-L2-L1-L1 ...) and a memory-based specific sequential order after hearing an auditory response-signal in each trial (i.e., producing sentences with the language, which had to be switched after every second word/trial). The word sequence was either a sentence that was syntactically correct in both languages and could be translated word-for-word into the other language (language-unspecific sentence), a sentence that was correct in just one language and not in a word-for-word translation into the other language (language-specific sentence) or a sentence that was syntactically incorrect in both languages (scrambled sentence). The results showed that the switch costs were smaller in the language-unspecific sentences than in the language-specific and scrambled sentences, which indicated an important role of the sentential context for language switch costs. The researchers suggested that the results could have arisen from the fact that less conflicting interference occurs within a languageunspecific sentence because it was syntactically correct in both languages. Similarly, in another study, Gollan and Goldrick (2016) asked Spanish-English bilinguals to read aloud paragraphs of texts containing mostly grammatical language switches (e.g., switches that conform to the naturally occurring constraints of both languages) or mostly ungrammatical (haphazard mixing) language switches. The results suggested that bilinguals read paragraphs with ungrammatical switches more slowly and produced more intrusion errors than when reading 
paragraphs with grammatical language switches (Gollan \& Goldrick, 2016). One possible explanation is that compared to paragraphs with grammatical switches, paragraphs with ungrammatical switches induced a higher conflicting reading context due to increased interference. These accounts would be consistent with the adaptive control hypothesis, which postulates that the interactive contexts of a bilingual conversation place different levels of demand on the cognitive system and its neural mechanisms and thus alter their various control abilities (Abutalebi \& Green, 2016; Green \& Abutalebi, 2013). Specifically, this hypothesis suggests that processing contexts with different demands on cognitive control resources would affect the language control system differently. The findings of several recent studies also support the idea that the processing context is a critical factor that modulates bilingual language control (Hartanto \& Yang, 2016; Olson, 2016; Ye, Mo \& Wu, 2016; Zhang, Huang, Song, Fang, Shen, Li, Gong \& Xie, 2014).

In the present study, we explore the extent to which the processing context affects the switch costs in bilinguals. Two experiments are conducted. In Experiment 1, non-proficient Chinese-English bilinguals performed a modified language-switching task in which the context was either conflicting (naming the ink colour of colour words in a switch task) or non-conflicting (naming the ink colour of non-colour words in a switch task). Since domain-general cognitive control (i.e., the Stroop effect) and language control (i.e., the language switch cost) may share the same inhibitory mechanisms (Declerck et al., 2017; Prior \& Gollan, 2013), the need to resolve Stroop effect-related interference may reduce the availability of inhibitory control for language switching. Therefore, we predict that the switch costs are larger in the conflicting context than the non-conflicting context because there is less inhibitory control to deploy during language switching, given that a part of it has to be deployed to resolve the Stroop effect-related interference. Furthermore, because L2-L1 switches require greater cognitive processing resources than L1-L2 switches according to the ICM (Green, 1998), we proposed that the decrease in cognitive control resources would exert a greater influence on L2-L1 switch costs (i.e., the more difficult task) than on L1-L2 switch costs (i.e., the easier task). Thus, we predict that the level of asymmetry in switch costs is greater in the conflicting context than in the non-conflicting context. In Experiment 2, we examine the hypothesis that the capacities of domaingeneral cognitive control affect language switch costs by dividing the participants into high-IC and low-IC groups based on their performance in a non-linguistic cognitive control task (i.e., the spatial Stroop task). Our prediction is that the participants in the low-IC group would show a larger asymmetry of switch costs than the high-IC group, between L1-L2 and L2-L1 switches (e.g., Liu et al.,
Table 1. Means and SDs of the AoA and proficiency ratings in four language skills for both Chinese and English.

\begin{tabular}{lll}
\hline \hline Self-ratings & L1 (Chinese) & L2 (English) \\
\hline AOA & & $8.61(2.43)$ \\
Listening & $7.64(2.23)$ & $4.21(2.04)$ \\
Speaking & $7.25(1.91)$ & $4.04(1.85)$ \\
Reading & $7.21(1.52)$ & $5.04(1.93)$ \\
Writing & $6.71(1.94)$ & $4.64(1.93)$ \\
\hline \hline
\end{tabular}

2014), because the individual capacity of cognitive control would exert a greater influence on the more difficult task (L2-L1 switch) than on the easier task (L1-L2 switch). Moreover, critically, we aimed to examine whether the individual capacity of cognitive control modulates the influence of the processing context on language switch costs. Specifically, if individual variances in cognitive control capacities modulate the processing context effects on bilingual switch costs, then manipulations of the two variables would lead to an interaction. In contrast, the absence of such an interaction would imply that the processing context and cognitive control capacities influence bilingual language control independently.

\section{Experiment 1}

\subsection{Participants}

Thirty undergraduate students from the South China Normal University were paid to participate in the experiment, which had been approved by the ethical committee of the local authority. All participants were right-handed with normal or corrected-to-normal vision, and they signed a written informed consent form prior to their participation. The participants were non-English major students, and their mean age of acquisition (AoA) was $8.61( \pm 2.43)$ for English. The participants rated their proficiency level in L1 (Chinese) and L2 (English) for listening, speaking, reading, and writing on an elevenpoint Likert scale, with 10 indicating the highest level of proficiency and 0 indicating the lowest level of proficiency. Paired-samples $t$ tests revealed a significant difference between the proficiency ratings in L1 and L 2 for all four language skills (all $t s>5.2$, all $p s<0.001$ ), suggesting that the participants were unbalanced bilinguals with a higher proficiency level in L1 than in L2 (see Table 1).

\subsection{Task and procedure}

The experimental task combines a language switching manipulation with the classic colour-word Stroop paradigm. The experimental materials were 3 colour 


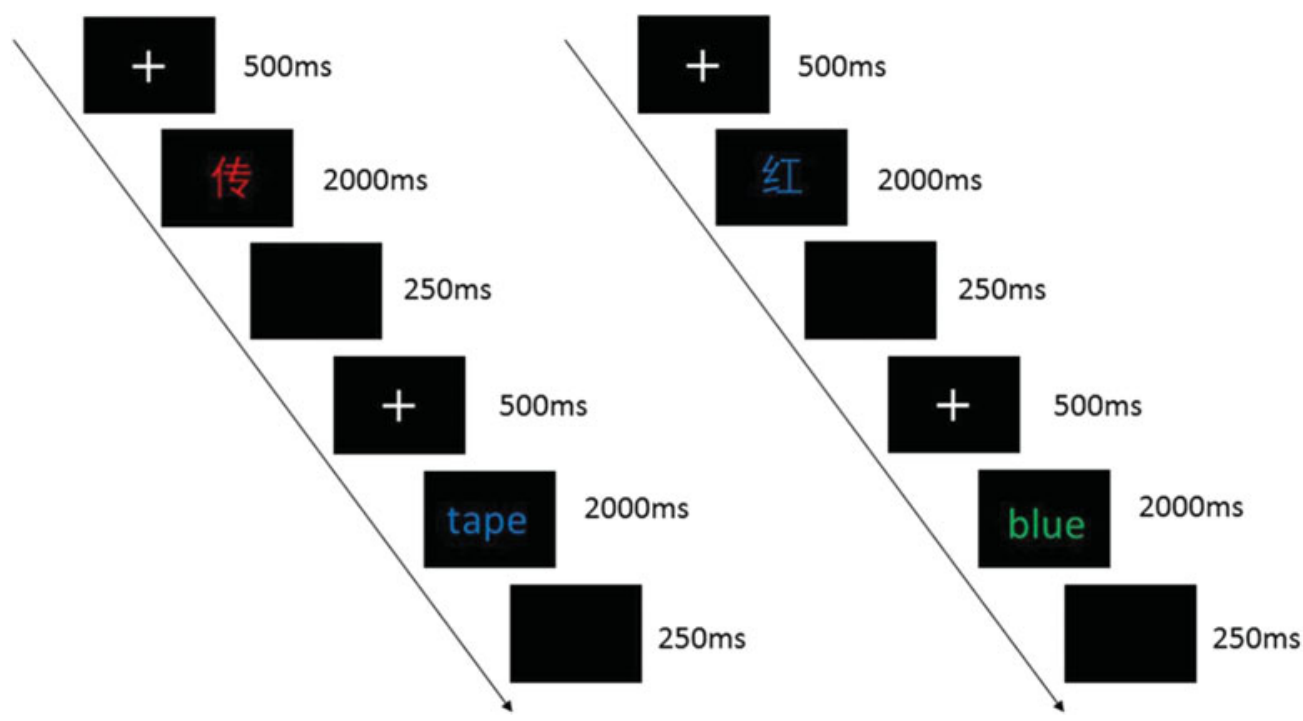

Figure 1. (Colour online) The unfolding of language switching naming trials in the non-Stroop (left panel) and the Stroop (right panel) blocks. Each trial began with a fixation cross that lasted for $500 \mathrm{~ms}$. Then, the stimulus was presented at the same location as the fixation cross and lasted until the response was made and no longer than $2000 \mathrm{~ms}$. The inter-trial interval was $250 \mathrm{~ms}$.

words ("red", "green", and "blue") and 3 non-colour words ("tap", "carry", and "dive"), all matched on the number of letters in English and the number of strokes in their Chinese translations. The participants were instructed to name the colour of the printed Chinese words in Chinese and the colour of the printed English words in English. There were a conflicting block and a non-conflicting block of the task. In the conflicting block, colour words were presented in an inconsistent colour (e.g., the word "red" was presented either in green or blue), and participants were instructed to name the printed colour of the words. In the non-conflicting block, non-colour words were presented in any of the three colours (e.g., "tap" could be presented in red, green, or blue), and the participants were also instructed to name the printed colour of the non-colour words. The block order was appropriately counterbalanced across participants. Therefore, the experiment conforms to a 2 (block: conflicting versus non-conflicting) $\times 2$ (language: L1 versus L2) within-subjects design, with switch costs of RTs and error rates (switching trials-repeated trials) as the dependent variables. In both blocks, the ratio of switching trials and repeated trials was 1:1 for each language. Each block consisted of 121 experimental trials, with the first trial being the filler trial. Every trial began with a fixation cross presented at the centre of the screen for 500 milliseconds, followed by the presentation of a word for 2000 milliseconds or for as long as it took the participant to respond if it was within 2000 milliseconds (see Figure 1). A video recording software named "EV Capture" recorded the progress of the experiment, including the verbal responses made by the participants, which were checked for accuracy post-experiment.

\subsection{Results}

The first trial of each block and the error trials were excluded from RT analyses, as were trials following an error trial. We also discarded trials with RTs over 2.5 standard deviations (SDs) below or above the mean (per condition) (Wang, Fan, Liu \& Cai, 2016). Taking these criteria into account, a total of $9.4 \%$ of the data (ranging from $7.6 \%$ to $14.1 \%$ for different conditions) were excluded. Furthermore, two participants were excluded from the analysis due to an error rate higher than $20 \%$ (Heikoop, Declerck, Los \& Koch, 2016). The switch costs for the remaining participants were calculated by subtracting the reaction times and the accuracy rates in the repeated trials from the switch trials. We performed $2 \times 2$ repeated-measures ANOVA with language (L1 versus L2) and block (conflicting versus non-conflicting) as within-subject variables on reaction times (see Table 2 ). The results showed that the main effect of language is significant, $F(1,27)=18.226, p<0.001, \eta_{\mathrm{p}}^{2}=0.403$, indicating significantly larger switch costs in L1 than in L2. There was also a significant main effect of block, $F$ $(1,27)=5.235, p=0.030, \eta_{\mathrm{p}}^{2}=0.162$, indicating that the switch costs in the conflicting block are significantly larger than those in the non-conflicting block. There was also a significant interaction between language and block, $F(1,27)=5.436, p=0.027, \eta_{\mathrm{p}}^{2}=0.168$. Further pairedsamples $t$ tests revealed that the switch costs from L2 
Table 2. Mean RTs (ms) and SDs in the repeat and switch trials decomposed by language and processing context.

\begin{tabular}{lcccccccc}
\hline \hline & \multicolumn{3}{c}{ L1 } & & & \multicolumn{2}{c}{ L2 } & \\
\cline { 2 - 4 } & Repeat & switch & costs & & Repeat & switch & costs \\
\hline Non-colour word (non-conflicting) & $805(97)$ & $997(153)$ & $192(92)$ & & $877(103)$ & $1027(119)$ & $150(75)$ \\
Colour word (conflicting) & $1032(144)$ & $1303(237)$ & $271(163)$ & & $1046(124)$ & $1207(220)$ & $161(127)$ \\
\hline \hline
\end{tabular}

Table 3. Mean accuracy and SDs in the repeat and switch trials decomposed by language and processing context.

\begin{tabular}{|c|c|c|c|c|c|c|}
\hline & \multicolumn{3}{|c|}{ L1 } & \multicolumn{3}{|c|}{$\mathrm{L} 2$} \\
\hline & Repeat & switch & costs & Repeat & switch & costs \\
\hline Non-colour word (non-conflicting) & $.96(.05)$ & $.93(.07)$ & $-.03(.06)$ & $.95(.04)$ & $.92(.06)$ & $-.03(.05)$ \\
\hline Colour word (conflicting) & $.95(.06)$ & $.89(.08)$ & $-.06(.06)$ & $.95(.05)$ & $.89(.06)$ & $-.06(.05)$ \\
\hline
\end{tabular}

to L1 were significantly larger in the conflicting block than in the non-conflicting block (Diff $=79 \mathrm{~ms}: 192 \mathrm{~ms}$ vs. $271 \mathrm{~ms}), t(27)=3.246, p=0.003, d=1.249$. The switch costs from L1 to L2 were not significantly different between the two blocks (Diff $=11 \mathrm{~ms}: 150 \mathrm{~ms}$ vs. $161 \mathrm{~ms}$ ), $t(27)=0.437, p=0.666$. Critically, the switch costs in L1 were significantly larger than those in L2 in the conflicting block (Diff $=110 \mathrm{~ms}: 271 \mathrm{~ms}$ vs. $161 \mathrm{~ms}), t(27)=4.637$, $p<0.001, d=1.785$, but not the non-conflicting block (Diff $=42 \mathrm{~ms}: 192 \mathrm{~ms}$ vs. $150 \mathrm{~ms}$ ), $t(27)=1.871, p=$ $0.072, d=0.720$.

A repeated-measures ANOVA was performed on the accuracy rates (see Table 3). The results showed a significant main effect of block, $F(1,27)=9.567$, $p=0.005, \eta_{\mathrm{p}}^{2}=0.262$, indicating larger switch costs in the conflicting block than in the non-conflicting block. The main effect for language was not significant, $F(1,27)=$ $0.002, p=0.963$. The interaction between block and language was also not significant, $F(1,27)=0.001, p=$ 0.971 .

\subsection{Discussion}

The results of Experiment 1 showed that the conflicting context, induced by the colour-word Stroop, enhanced the magnitude of the switch costs in bilinguals compared to the non-conflicting context. This result suggests that the processing context can affect the language switch costs in bilinguals. We also observed an interaction between the processing context and the language, resulting in greater asymmetrical switch costs in the conflicting context than in the non-conflicting context. According to the ICM, bilinguals inhibit the activation level of the non-target language to facilitate the processing of the target language. Therefore, for non-proficient bilinguals, L2-L1 switches require greater cognitive processing resources than L1L2 switches because compared to the less dominant L2, the inhibition of the more dominant L1 consumes more resources, leading to increased difficulty reactivating L1 compared to L2 after the switch. Then, the processing context (non-conflicting vs. conflicting) exerted a greater influence on the more difficult task (i.e., L2-L1 switches) than the easier task (i.e., L1-L2 switches). Thus, the conflicting context should cause greater costs for L2-L1 than for L1-L2 or enhance the cost of L2-L1 but not L1L2 switches. Then, greater L1-L2 asymmetries would be found in conflicting vs. non-conflicting contexts. Taken together, our findings suggest that the processing context plays a critical role in modulating language switch costs.

While the findings of Experiment 1 showed that the processing context affects the language switch costs in bilinguals, Experiment 2 investigates whether the context effects can be modulated by individual variance in cognitive control capacities, given that previous studies have shown that individuals with different levels of IC showed different switch costs. To address this problem, in Experiment 2, the participants were first divided into highIC and low-IC groups based on their performance in a nonlinguistic, spatial Stroop task, before the administration of the language-switching task used in Experiment 1. This design allows Experiment 2 to verify the context effects observed in Experiment 1 and to examine the hypothesis that the effects of individual variances in cognitive control capacities and the effects of the processing context on bilingual switch costs share the same cognitive resources.

\section{Experiment 2}

\subsection{Participants}

Fifty undergraduate students from the South China Normal University were paid to participate in the experiment. All participants signed the written informed consent form, and the study was approved by the 
Table 4. Means and SDs of the AoA and proficiency ratings in four language skills for both Chinese and English.

\begin{tabular}{|c|c|c|c|c|c|c|c|c|}
\hline \multirow{2}{*}{$\begin{array}{l}\text { Self- } \\
\text { rating }\end{array}$} & \multicolumn{4}{|c|}{ L1(Chinese) } & \multicolumn{4}{|c|}{ L2 (English) } \\
\hline & High-IC & Low-IC & $t$ & $p$ & High-IC & Low-IC & $t$ & $p$ \\
\hline $\mathrm{AOA}$ & & & & & $8.79(1.91)$ & $8.17(1.66)$ & 1.21 & 0.23 \\
\hline Listening & $7.58(1.01)$ & $7.21(1.31)$ & 1.10 & 0.28 & $3.96(1.43)$ & $3.75(0.94)$ & 0.60 & 0.55 \\
\hline Speaking & $6.63(1.01)$ & $6.42(1.13)$ & 0.67 & 0.52 & $3.96(1.46)$ & $3.96(0.91)$ & 0.01 & 0.99 \\
\hline Reading & $7.21(1.38)$ & $6.88(1.32)$ & 0.85 & 0.40 & $5.29(1.68)$ & $5.00(1.14)$ & 0.70 & 0.49 \\
\hline Writing & $6.46(1.35)$ & $6.33(1.68)$ & 0.28 & 0.79 & $4.96(1.62)$ & $4.79(1.29)$ & 0.39 & 0.70 \\
\hline
\end{tabular}

local authority. The participants were right-handed, had normal or corrected-to-normal vision, and had no reported psychological conditions. They were non-English major students, and all completed the self-rating questionnaire used in Experiment 1. The mean proficiency ratings of L1 and L2 in listening, speaking, reading, and writing are shown in Table 4. Paired-samples $t$ tests revealed a significant difference between the proficiency ratings of L1 and L2 for all four skills (all $t s>7.2$, all $p s<$ .001 ), suggesting that the participants were unbalanced bilinguals with higher proficiency in L1 than in L2.

\subsection{Assessment of inhibitory control ability}

We used a spatial Stroop task as a measurement of the domain-general cognitive control ability. This task was adapted from the task used by Blumenfeld and Marian (2013), which has been widely considered the standard assessment in previous studies (Giezen, Blumenfeld, Shook, Marian \& Emmorey, 2015). In the spatial Stroop task, a black arrow is presented on the screen, and the participants are instructed to respond to the direction to which this arrow points by pressing keys with their left or right hand while ignoring its location (i.e., left side, right side, or centre of the screen). The direction and location of the arrow were manipulated to create neutral trials, congruent trials and incongruent trials. The neutral condition consisted of 42 trials with an arrow presented at the centre of the screen pointing to either the left or the right. The congruent condition consisted of 126 trials with an arrow pointing in the same direction as the side of the screen on which it is presented. The incongruent condition consisted of 42 trials with an arrow pointing in the opposite direction as the side of the screen on which it is presented. The direction of the arrow and its location were balanced between left and right for all conditions. The ratio of congruent to incongruent trials was $3: 1$ in order to increase the Stroop effect, which is calculated by subtracting the reaction times and the error rates of the neutral condition from the incongruent condition.

Each trial started with a $500 \mathrm{~ms}$ fixation cross presented at the centre of the screen, followed by the presentation of an arrow for $1200 \mathrm{~ms}$ or until the participants responded to it. The inter-trial interval was a $500 \mathrm{~ms}$ blank screen. The participants were instructed to press the "F" key for leftward-facing arrows and the "J" key for rightwardfacing arrows while ignoring the location of the arrow. The experiment began with 20 practice trials ( 4 neutral, 4 incongruent, 12 congruent), and the rest of the experiment was separated into two blocks by a brief pause. The presentation of the trials was pseudo-randomized such that there were no more than three consecutive left or right responses. Furthermore, there were 8 repeat trials and 34 switch trials in both the neutral and incongruent conditions. Twenty-eight of these switch trials involved a switch from a congruent trial to a neutral or incongruent trial. In the remaining six switch trials, half were switching from neutral trials to incongruent trials, and the other half were switching from incongruent trials to neutral trials.

\subsection{The high-IC group and the low-IC group}

The mean accuracy rate and mean response latencies are shown in Table 5. The participants were split into the high-IC group and the low-IC group in terms of response latencies but not accuracy rates, as the latter did not sufficiently categorize the participants into high and low-IC groups because many participants had a similar level of accuracy. The top 25 participants formed the high-IC group, and the bottom 25 participants formed the low-IC group. An independent-samples $t$-test on the mean response latencies showed a significant difference between both groups, $t(47)=6.333, p<0.001$; a similar result was found for the accuracy rates, $t(47)=1.935, p=$ 0.059 . In addition, we conducted a statistical analysis on the performance of the high-IC and low-IC participants in all three conditions of the Stroop task independently and found a significant between-group difference in the accuracy rates for the incongruent condition $(t(47)=$ 2.432, $p=0.019)$ but not for the congruent $(t(47)=$ $0.831, p=0.410)$ or neutral conditions $(t(47)=1.063, p=$ $0.293)$. Similarly, we found a nearly significant betweengroup difference in the response latencies $(t(47)=1.647$, $p=0.106)$ but not for the congruent $(t(47)=0.202$, 
Table 5. Mean RTs (ms) and ACCs in the neutral, congruent, and incongruent condition for the high-IC and low-IC groups in the spatial Stroop task.

\begin{tabular}{lccllc}
\hline \hline & & Neutral & Congruent & Incongruent & Stroop effect \\
\hline RT & High-IC & $426(47)$ & $431(48)$ & $498(55)$ & $72(20)$ \\
& Low-IC & $414(43)$ & $428(57)$ & $523(54)$ & $109(20)$ \\
\multirow{2}{*}{ ACC } & High-IC & $.99(.02)$ & $.98(.02)$ & $.86(.08)$ & $-.13(.08)$ \\
& Low-IC & $.95(.19)$ & $.95(.19)$ & $.76(.19)$ & $-.18(.13)$ \\
\hline \hline
\end{tabular}

$p=0.840)$ or neutral conditions $(t(47)=862, p=$ $0.393)$. These results provided more confidence that the distinction was psychologically real.

Table 4 shows the AoA of L2 and the ratings of four language skills obtained through the self-rating questionnaire. Independent-samples $t$ tests showed no significant difference between the high-IC and low-IC participants in L2 AoA of the language skill ratings for both Chinese and English (all ps > 0.1).

\subsection{Task and procedure}

Experiment 2 conforms to a 2 (group: high-IC versus low-IC) $\times 2$ (block: conflicting versus non-conflicting) $\times$ 2 (language: L1 versus L2), with group as the betweensubject variable and block and language as within-subject variables. The dependent variables were switch costs of RTs and error rates. The modified language switching naming task used in Experiment 2 was the same as that used in Experiment 1. A video recording software named "EV Capture" recorded the visual stimulus presentation and the verbal responses of the participants, which were checked for accuracy post-experiment.

\subsection{Results}

We used outlier criteria and error definitions identical to those in Experiment 1, which resulted in the exclusion of $8.3 \%$ of the data (ranging from $6.9 \%$ to $12.3 \%$ for different conditions). One participant was excluded from analysis for having an error rate higher than $20 \%$. The mean RTs are presented in Table 6.

The switch costs were computed by subtracting the RTs for the repeat condition from the switch condition. A 2 (language: L1 versus L2) $\times 2$ (block: conflicting versus non-conflicting) $\times 2$ (group: high-IC vs. lowIC) repeated-measures ANOVA was conducted on the reaction times. The results showed a significant main effect of language, $F(1,47)=24.921, p<0.001$, $\eta_{\mathrm{p}}^{2}=0.347$, suggesting that L2-L1 switch costs are significantly larger than L1-L2 switch costs. There was also a significant main effect of block, $F(1,47)=22.592$, $p<0.001, \eta_{\mathrm{p}}^{2}=0.325$, indicating that the switch costs in the conflicting block are significantly larger than those in the non-conflicting block. In addition, we found a significant main effect of group, $F(1,47)=5.910, p=$ $0.019, \eta_{\mathrm{p}}^{2}=0.112$, suggesting significantly larger switch costs for the low-IC group than for the high-IC group. Moreover, we found a marginally significant interaction between language and block, $F(1,47)=3.236, p=$ $0.078, \eta_{\mathrm{p}}^{2}=0.064$. Paired-samples $t$ tests revealed that the L2-L1 switch costs are slightly larger than the L1L2 switch costs in the non-conflicting block (Diff $=33$ ms: $155 \mathrm{~ms}$ vs. $122 \mathrm{~ms}), t(47)=2.699, p=0.010$, $d=1.039$, while the $\mathrm{L} 2-\mathrm{L} 1$ switch costs are significantly larger than the L1-L2 switch costs in the conflicting block (Diff $=76 \mathrm{~ms}: 242 \mathrm{~ms}$ vs. $166 \mathrm{~ms}$ ), $t(47)=3.914, p$ $<0.001, d=1.506$. In addition, we found no significant interaction effect between block and group, $F(1,47)=$ $1.687, p=0.200$, and no significant interaction effect between language and group, $\mathrm{F}(1,47)=0.287, p=0.595$. Critically, however, the three-way interaction effect among language, block and group was marginally significant, $\mathrm{F}$ $(1,47)=3.985, p=0.052, \eta_{\mathrm{p}}^{2}=0.078$. Further analysis showed that the interaction between block and language was significant for the high-IC participants, $\mathrm{F}(1,24)=$ $11.48, p=0.002, \eta_{\mathrm{p}}^{2}=0.324$, but not for the low-IC participants, $\mathrm{F}(1,23)=0.01, p=0.907$. Paired-samples $t$ tests revealed that for the high-IC participants, the L2-L1 switch costs are similar to the L1-L2 switch costs in the non-conflicting block (Diff $=16 \mathrm{~ms}: 134 \mathrm{~ms}$ vs. $118 \mathrm{~ms}$ ), $t(24)=0.804, p=0.429$, while the L2-L1 switch costs are significantly larger than the L1-L2 switch costs in the conflicting block (Diff $=105 \mathrm{~ms}: 226 \mathrm{~ms}$ vs. $121 \mathrm{~ms}$ ), $t$ $(24)=5.046, p<0.001, d=1.942$. Additionally, to better illustrate the group differences (high-IC participants vs. low-IC participants) in different contexts (non-conflicting context vs. conflicting context), we computed the switch costs asymmetry as the L1 switch costs - the L2 switch costs first. Then independent-samples $t$ tests revealed that compared to the low-IC participants, the high-IC participants showed a numerically smaller asymmetry of switch costs in the non-conflicting context (Diff $=35$ ms: $16 \mathrm{~ms}$ vs. $51 \mathrm{~ms}), t(47)=1.461, p=0.151$, and a numerically larger asymmetry of switch costs in the conflicting context (Diff $=59 \mathrm{~ms}: 105 \mathrm{~ms}$ vs. $46 \mathrm{~ms}$ ), $t$ $(47)=1.526, p=0.134$, though these differences did not reach significance. 


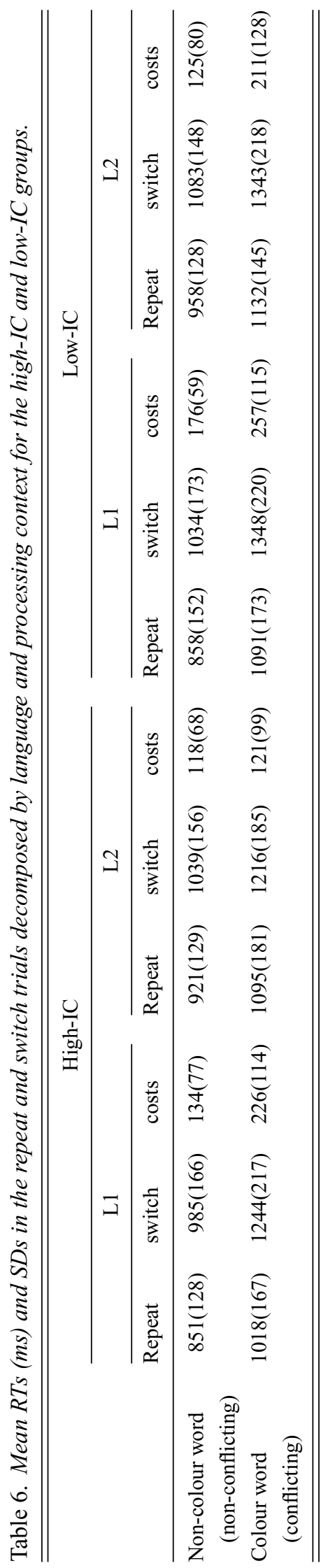

A repeated-measures ANOVA was performed on the accuracy rates (see Table 7). The results showed that all the main effects and interaction effects were not significant ( $p s>0.05$ ). However, there was a marginally significant interaction between block and the switch direction, $F(1$, $47)=3.548, p=0.066, \eta_{\mathrm{p}}^{2}=0.070$. Paired-samples $t$ tests revealed that the L2-L1 switch costs are comparable to the L1-L2 switch costs in the non-conflicting block (Diff $=$ 0.005 : -0.030 vs. -0.035$), t(47)=0.668, p=0.507$, while the L2-L1 switch costs are marginally significantly larger than the L1-L2 switch costs in the conflicting block $($ Diff $=0.020:-0.055$ vs. -0.035$), t(47)=1.908, p=$ $0.062, d=0.779$.

In addition, we performed a correlation analysis between the spatial Stroop task and the language switch task. The results showed a marginally significant positive correlation between the magnitude of the spatial Stroop effect and the L1 switch costs, $\mathrm{r}(49)=0.242, p=0.094$, but no correlation with the L2 switch costs, $\mathrm{r}(49)=$ $0.226, p=0.118$, though the results of these correlations are very similar. We also correlated the magnitude of the spatial Stroop effect with the difference between the L1 and L2 switch costs (a measure of the asymmetry). The results additionally showed a marginally significant positive relation, $\mathrm{r}(49)=0.272, p=0.058$.

\subsection{Discussion}

The findings of Experiment 2 replicated the context effect that was found in Experiment 1: the conflicting context enhanced the magnitude of the switch costs in bilinguals compared to the non-conflicting context. In addition, individuals with high IC showed overall smaller switch costs than those with low IC, a pattern of results that is consistent with previous studies (Linck et al., 2012; Liu, Rossi, Zhou \& Chen, 2014). Critically, however, contrary to our predictions, the participants in the low-IC group did not show overall larger switch cost asymmetries than those in the high-IC group. This finding is due to a particularly high value of the L2 switch cost in the conflicting condition for the low-IC participants (211 $\mathrm{ms})$ compared to the high-IC participants $(121 \mathrm{~ms})$. This result suggests that the conflicting context saturated the cognitive resources to a greater extent for the lowIC compared to the high-IC participants. In the nonconflicting context, the switch cost asymmetry is larger in the low-IC (176 ms vs. $125 \mathrm{~ms}$ ) than in the high-IC group (134 ms vs. $118 \mathrm{~ms})$. Although the difference was not significant, it is in the same direction as in Liu et al. (2014, 2016), who used a non-conflicting picture naming task. Taken together, these findings suggest that the processing context and the individual capacity of cognitive control affect the language switch costs in bilinguals and that, more critically, individual cognitive control capacity could modulate the context effect on switch costs. 


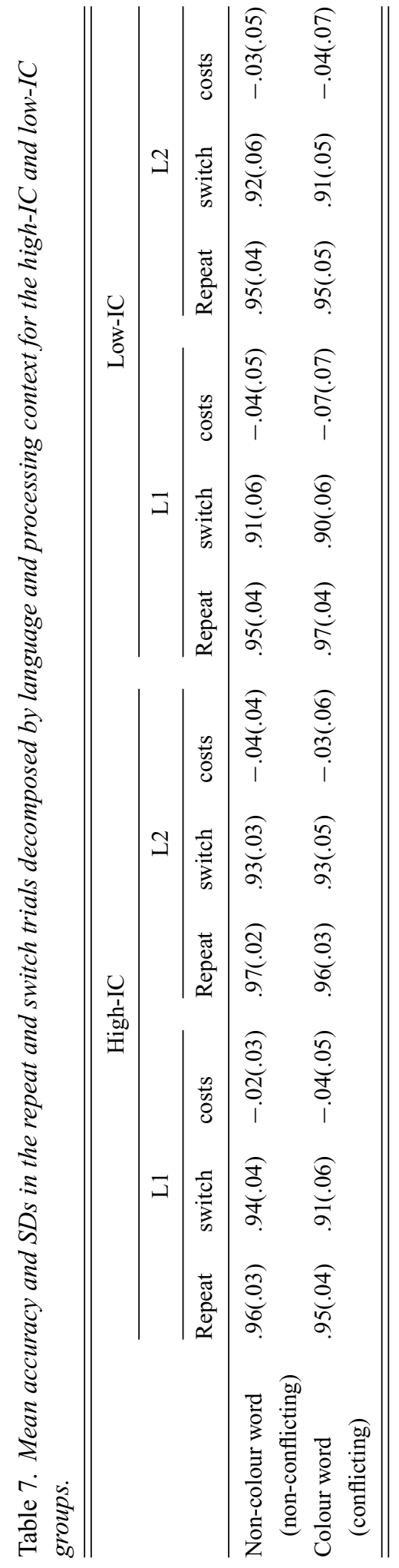

\section{General discussion}

In the present study, we investigated whether the language switch costs in bilingual speakers are influenced by the processing context and individual variances in domain-general cognitive control capacity. ChineseEnglish bilinguals performed a language-switching task in a conflicting and a non-conflicting context. The results from Experiment 1 and 2 both showed larger switch costs and greater asymmetrical switch costs in the conflicting context than in the non-conflicting context. Meanwhile, the results also showed that individuals with low capacity of cognitive control showed larger switch costs than that with high capacity of cognitive control. Furthermore, we found that the processing context and the individual capacity of cognitive control modulated interactively on bilingual language switch costs.

\subsection{The influence of a processing context on language switch costs}

Previous studies have shown that some task-dependent factors, such as the sentence context or grammatical structure, could modulate language switch costs (Declerck \& Philipp, 2015b; Gollan \& Goldrick, 2016). However, the potential cause for why task-dependent factors modulate language switch costs remains unclear. By analysing and summarizing the existing related studies, we speculated that the processing context might be a potential cause. To verify our hypothesis, the present study examined the impact of the processing context on language switch costs by using a Stroop task during a language switching paradigm, instead of the commonly used cued languageswitching task. Because a part of inhibitory control has to be deployed to solve the Stroop effect-related interference in the conflicting context, there is less inhibitory control to deploy during language switching, resulting in larger switch costs in the conflicting context than in the nonconflicting context. The conflict monitor hypothesis can also account for this finding (Botvinick, Braver, Branch, Carter \& Cohen, 2001). The conflicting context causes the larger switch costs because both language switching and the Stroop effect produce more conflict than in the non-conflicting context with only language switching. In addition, as L2-L1 switches require greater cognitive processing resources than L1-L2 switches for nonproficient bilinguals in language switching, the processing context would exert a greater influence on the more difficult task (i.e., L2-L1 switch) than the easier task (i.e., L1-L2 switch); thus, the level of asymmetry in switch costs is greater in the conflicting context than in the nonconflicting context. Both results suggest a critical role of the processing context for the size of language switch costs. 
The findings of our study demonstrated that the processing context with different degrees of interference could account for any task-dependent factors modulating language switch costs. For example, in addition to the sentence context and grammatical structure that we mentioned in the introduction, several studies have also shown that the symmetry of switch costs can be affected by the language-switching paradigms. Specifically, under certain conditions, including the sequence-based language-switching paradigm with predictable responses (Declerck, Koch \& Philipp, 2015; Declerck et al., 2013) and the voluntary non-cued language-switching paradigm with full freedom to switch (Gollan \& Ferreira, 2009; Kleinman \& Gollan, 2016), unbalanced bilinguals can show symmetrical switch costs (for a review, see Bobb \& Wodniecka, 2013). With regard to such results, we argued that perhaps just because both the sequence-based language switching paradigm and the voluntary noncued language switching paradigm have less processing interference than the classical cued language switching paradigm, speakers do not need to engage in much inhibition for L1 and L2 in bilingual switching. Thus, they exhibited symmetrical switch costs. In addition, previous language switching studies showed that switch cost asymmetries can be affected by the preparation time and that with a longer preparation time, switch cost asymmetries can be overcome (Ma, Li \& Guo, 2016; Verhoef et al., 2009). For such findings, it is easy to understand that a longer preparation time facilitated the conflict resolution in the processing context.

Moreover, our findings supported and expanded the adaptive control hypothesis, in which contexts modulate bilingual language control (Green \& Abutalebi, 2013). The adaptive control hypothesis proposed that linguistic experience with different interactional contexts of conversational exchanges places different level of demand on the brain and cognitive systems and adaptively alters their language control. The present study (both Experiment 1 and Experiment 2) investigated the performance of bilinguals in different nonlinguistic processing contexts (non-conflicting context or conflicting context) and observed that the switch costs and the level of symmetry in switch costs are significantly larger in the conflicting context than in the non-conflicting context. We proposed that in addition to the linguistic context, the non-linguistic context should also be added to the adaptive control hypothesis.

\subsection{The processing context and the individual capacity of cognitive control modulated interactively on bilingual language switch costs}

In recent years, studies have indicated that the individual capacity of cognitive control was a new factor that can influence language-switching performance (Liu et al.,
2016; Liu et al., 2015). The results of Experiment 2 in the current study were in line with such previous findings. In Experiment 2, we recruited nonproficient Chinese-English bilinguals with different capacities of inhibitory control (high IC or low IC) to complete a modified Stroop language-switching task in different processing contexts (non-conflicting context or conflicting context). The results showed that the highIC group exhibited smaller switch costs than the low-IC group, which demonstrated that the individual capacity of cognitive control could influence language switch costs.

Furthermore, we found that the individual capacity of cognitive control could modulate the influence of the processing context on language switch costs. In the current study, we observed an interaction between our conflicting manipulation and individual variances in cognitive control in Experiment 2. This result was inconsistent with the adaptive control hypothesis (Green \& Abutalebi, 2013), which indicated that individual variances such as the capacity of cognitive control might modulate the influence of the interactional context on a variety of control processes. However, not all related studies concluded so. For example, Gollan and Goldrick (2016) asked Spanish-English bilinguals to read aloud paragraphs that had different grammatical contexts, i.e., mostly grammatical (conforming to naturally occurring constraints) or mostly ungrammatical (haphazard mixing) language switches. They finally found no significant interactions between their grammaticality manipulation and individual variances in executive function. Such inconsistent findings might arise from the different types of contexts used in each study. To better examine how the individual capacity of cognitive control modulates different linguistic or non-linguistic contexts, researchers should conduct further investigations.

In addition, we also found that the processing context could modulate the influence of the individual capacity of cognitive control on language switch costs. Compared to the low-IC participants, the high-IC participants showed a slightly larger asymmetry of language switch costs in the non-conflicting context and a slightly smaller asymmetry of language switch costs in the conflicting context. This pattern of switch cost asymmetry in the non-conflicting context is in line with Liu et al. (2014), which has also used a task with less conflict. By contrast, the inconsistent results between the pattern of switch cost asymmetry in the conflicting context and previous studies (e.g., Liu et al., 2014) may be because the conflicting context brings much more conflict.

Overall, the current study indicated that the processing context and the individual capacity of cognitive control have an impact on language switch costs and that, more critically, both of them modulated interactively on bilingual language switch costs. 


\subsection{Interactions of bilingual language control and domain-general cognitive control}

Whether bilingual language control shared mechanisms with domain-general cognitive control has been debated for several years. Recent behavioural research on the overlap between bilingual language control and domain-general cognitive control has mainly correlated participants' behaviour in comparable tasks involving either bilingual language control or domain-general cognitive control processes. Some studies suggested that there is an overlap between language control and domaingeneral control (e.g., Declerck et al., 2017; Stasenko, Matt \& Gollan, 2017; Prior \& Gollan, 2013), while others indicated that there is no correlation between linguistic and non-linguistic switch costs (e.g., Calabria, Hernandez, Branzi \& Costa, 2011; Cattaneo, Calabria, Marne, Gironell, Abutalebi \& Costa, 2015; Calabria, Branzi, Marne, Hernandez \& Costa, 2015; Branzi, Calabria, Boscarino \& Costa, 2016). In addition, although neuroimaging studies have revealed an overlap between bilingual language control and domain-general cognitive control (e.g., Branzi, Della Rosa, Canini, Costa \& Abutalebi, 2015; Blanco-Elorrieta \& Pylkkänen, 2016), such an overlap was only partial in limited brain areas.

To address this controversy, the current study used a Stroop task during a language-switching paradigm. The results in both Experiment 1 and Experiment 2 showed that the conflicting context, as induced by the colour-word Stroop paradigm, enhanced the magnitude of the switch costs in bilinguals compared to the nonconflicting context, suggesting that cognitive resources are shared between the mechanisms involved in bilingual language control and domain-general cognitive control. Furthermore, the results of the behavioural correlation between the spatial Stroop task and the language switch task in Experiment 2 suggested that better inhibitors in the Stroop task were also better inhibitors in the language-switching task. Overall, our study supported the interactions of bilingual language control and domain-general cognitive control from two different perspectives.

\subsection{Theoretical implications}

The ICM, one prevailing theory about bilingual language control, proposed the important role of language proficiency in modulating language switch costs. It assumes that the degree of inhibition for language is positively correlated with language proficiency and that the releasing of a suppressed language is positively correlated with the degree of inhibition (Green, 1998). The classical evidence supporting the argument of the ICM was the finding that non-proficient bilinguals exhibited asymmetrical switch costs (Verhoef et al., 2009; Verhoef et al., 2010). One explanation for this finding was that the more dominant language needs to recruit more laborious inhibition as well as release from inhibition.

More recently, researchers have posited that the individual capacity of cognitive control also plays an analogous role in modulating language switch costs (Liu et al., 2015, 2016). Liu et al. (2015) found that the language switch costs of non-proficient bilinguals with high IC were symmetrical while that of nonproficient bilinguals with low IC were not. Their findings supplemented the IC model from a different perspective, implying not only that inhibition may play an important role during language switching but also that bilinguals with low proficiency can switch effectively between different languages if their capacity of inhibitory control is greatly enhanced.

While previous results have demonstrated individual variability in inhibition driven by proficiency or cognitive control ability, the current study suggests an interpretation of individual variability in inhibition a perspective of processing contexts (i.e., conflicting versus nonconflicting). The switch costs in the non-conflicting context were different from those in the conflicting context, implying different amounts of inhibition has been induced by each processing context. As described by the ICM framework, switch costs result from the amount of inhibition being applied to each language; then, according to the adaptive control hypothesis (Green \& Abutalebi, 2013), switching languages in different contexts would entail different levels of inhibition. The larger switch costs found in the conflicting as compared to the non-conflicting context may be due to reduced inhibitory control resources by the Stroop effect-related interference in the conflicting, but not non-conflicting, context.

In summary, in addition to language proficiency and individual capacity of cognitive control, two factors that have been shown to affect language switch costs, we demonstrated that the processing context plays an analogous role in modulating language-switching costs. We therefore suggest that both linguistic and nonlinguistic contexts should be incorporated into the ICM framework.

\section{Conclusion}

The present study reveals for the first time that there are different patterns of language switch costs in conflicting versus non-conflicting contexts. This finding suggests the critical role of the processing context in bilingual language switching. In addition, the observed interaction between the individual capacity of cognitive control and the processing context suggests that the processing context and the individual capacity of cognitive control modulated interactively on bilingual language switch costs. 


\section{References}

Abutalebi, J., \& Green, D. W. (2016). Neuroimaging of language control in bilinguals: neural adaptation and reserve. Bilingualism: Language and Cognition, 19(4), 689698.

Blanco-Elorrieta, E., \& Pylkkänen, L. (2016). Bilingual language control in perception versus action: MEG reveals comprehension control mechanisms in anterior cingulatecortex and domain-general control of production in dorsolateral prefrontal cortex. The Journal of Neuroscience, 36(2), 290-301

Blumenfeld, H. K., \& Marian, V. (2013). Parallel language activation and cognitive control during spoken word recognition in bilinguals. Journal of Cognitive Psychology, 25(5), 547-567.

Bobb, S. C., \& Wodniecka, Z. (2013). Language switching in picture naming: What asymmetric switch costs (do not) tell us about inhibition in bilingual speech planning. Journal of Cognitive Psychology, 25(5), 568-585.

Botvinick, M. M., Braver, T. S., Barch, D. M., Carter, C. S., \& Cohen, J. D. (2001). Conflict monitoring and cognitive control. Psychological Review, 108, 624-652.

Branzi, F. M., Calabria, M., Boscarino, M. L., \& Costa, A. (2016). On the overlap between bilingual language control and domain-general executive control. Acta psychologica, 166, 21-30.

Branzi, F. M., Della Rosa, P. A., Canini, M., Costa, A., \& Abutalebi, J. (2015). Language control in bilinguals: Monitoring and response selection. Cerebral Cortex, 26(6), 2367-2380.

Calabria, M., Branzi, F. M., Marne, P., Hernandez, M., \& Costa, A. (2015). Age-related effects over bilingual language control and executive control. Bilingualism: Language and Cognition, 18(1), 65-78.

Calabria, M., Hernández, M., Branzi, F. M., \& Costa, A. (2011). Qualitative differences between bilingual language control and executive control: Evidence from task-switching. Frontiers in psychology, 2, 399-408.

Cattaneo, G., Calabria, M., Marne, P., Gironell, A., Abutalebi, J., \& Costa, A. (2015). The role of executive control in bilingual language production: A study with Parkinson's disease individuals. Neuropsychologia, 66, 99-110.

Chang, S., Xie, J., Li, L., Wang, R., \& Liu, M. (2016). Switch costs occur at lemma stage when bilinguals name digits: evidence from language-switching and event-related potentials. Frontiers in Psychology, 7, 1249.

Costa, A., \& Santesteban, M. (2004). Lexical access in bilingual speech production: Evidence from language switching in highly proficient bilinguals and L2 learners. Journal of Memory and Language, 50(4), 491-511.

Costa, A., Santesteban, M., \& Caño, A. (2005). On the facilitatory effects of cognate words in bilingual speech production. Brain and Language, 94(1), 94-103.

Costa, A., Santesteban, M., \& Ivanova, I. (2006). How do highly proficient bilinguals control their lexicalization process? Inhibitory and language-specific selection mechanisms are both functional. Journal of Experimental Psychology: Learning, Memory, and Cognition, 32(5), 1057-1074. de Bruin, A., Roelofs, A., Dijkstra, T., \& FitzPatrick, I. (2014). Domain-general inhibition areas of the brain are involved in language switching: FMRI evidence from trilingual speakers. Neuroimage, 90, 348-359.

Declerck, M., Koch, I., \& Philipp, A. M. (2015). The minimum requirements of language control: Evidence from sequential predictability effects in language switching. Journal of Experimental Psychology: Learning, Memory, and Cognition, 41(2), 377-394.

Declerck, M., Grainger, J., Koch, I., \& Philipp, A. M. (2017). Is language control just a form of executive control? Evidence for overlapping processes in language switching and task switching. Journal of Memory and Language, 95, 138-145.

Declerck, M., \& Philipp, A. M. (2015a). A review of control processes and their locus in language switching. Psychonomic bulletin \& review, 22(6), 1630-1645.

Declerck, M., \& Philipp, A. M. (2015b). A sentence to remember: Instructed language switching in sentence production. Cognition, 137, 166-173.

Declerck, M., Philipp, A. M., \& Koch, I. (2013). Bilingual control: Sequential memory in language switching. Journal of Experimental Psychology: Learning, Memory, and Cognition, 39(6), 1793-1806.

Hernandez, A. E., Dapretto, M., Mazziotta, J., \& Bookheimer, S. (2001). Language switching and language representation in Spanish-English bilinguals: An fMRI study. Neuroimage, 14(2), 510-520.

Giezen, M. R., Blumenfeld, H. K., Shook, A., Marian, V., \& Emmorey, K. (2015). Parallel language activation and inhibitory control in bimodal bilinguals. Cognition, 141, 9-25.

Gollan, T. H., \& Ferreira, V. S. (2009). Should I stay or should I switch? A cost-benefit analysis of voluntary language switching in young and aging bilinguals. Journal of Experimental Psychology: Learning, Memory, and Cognition, 35(3), 640-665.

Gollan, T. H., \& Goldrick, M. (2016). Grammatical constraints on language switching: Language control is not just executive control. Journal of Memory and Language, 90, 177-199.

Gollan, T. H., Kleinman, D., \& Wierenga, C. E. (2014). What's easier: doing what you want, or being told what to do? Cued versus voluntary language and task switching. Journal of Experimental Psychology: General, 143(6), 21672195.

Green, D. W. (1998). Mental control of the bilingual lexicosemantic system. Bilingualism: Language and Cognition, 1(02), 67-81.

Green, D. W., \& Abutalebi, J. (2013). Language control in bilinguals: The adaptive control hypothesis. Journal of Cognitive Psychology, 25(5), 515-530.

Guo, T., Liu, F., Chen, B., \& Li, S. (2013). Inhibition of non-target languages in multilingual word production: Evidence from Uighur-Chinese-English trilinguals. Acta Psychologica, 143(3), 277-283.

Hartanto, A., \& Yang, H. (2016). Disparate bilingual experiences modulate task-switching advantages: A diffusion-model analysis of the effects of interactional context on switch costs. Cognition, 150, 10-19. 
Heikoop, K. W., Declerck, M., Los, S. A., \& Koch, I. (2016). Dissociating language-switch costs from cue-switch costs in bilingual language switching. Bilingualism: Language and Cognition, 19(05), 921-927.

Kleinman, D., \& Gollan, T. H. (2016). Speaking Two Languages for the Price of One: Bypassing Language Control Mechanisms via Accessibility-Driven Switches. Psychological Science, 27(5), 700-714.

Kroll, J. F., Bobb, S. C., Misra, M., \& Guo, T. (2008). Language selection in bilingual speech: Evidence for inhibitory processes. Acta Psychologica, 128(3), 416-430.

Linck, J. A., Schwieter, J. W., \& Sunderman, G. (2012). Inhibitory control predicts language switching performance in trilingual speech production. Bilingualism: Language and Cognition, 15(3), 651-662.

Liu, H., Liang, L., Dunlap, S., Fan, N., \& Chen, B. (2016). The effect of domain-general inhibition-related training on language switching: An ERP study. Cognition, 146, 264276.

Liu, H., Liang, L., Zhang, L., Lu, Y., \& Chen, B. (2015). Modulatory role of inhibition during language switching: Evidence from evoked and induced oscillatory activity. International Journal of Bilingualism, 21(1), 57-80.

Liu, H., Rossi, S., Zhou, H., \& Chen, B. (2014). Electrophysiological evidence for domain-general inhibitory control during bilingual language switching. PLoS One, 9(10), e110887.

Ma, F., Li, S., \& Guo, T. (2016). Reactive and proactive control in bilingual word production: An investigation of influential factors. Journal of Memory and Language, 86, 35-59.

Meuter, R. F., \& Allport, A. (1999). Bilingual language switching in naming: Asymmetrical costs of language selection. Journal of Memory and Language, 40(1), 2540.

Olson, D. J. (2016). The gradient effect of context on language switching and lexical access in bilingual production. Applied Psycholinguistics, 37(3), 725-756.
Prior, A., \& Gollan, T. H. (2013). The elusive link between language control and executive control: A case of limited transfer. Journal of Cognitive Psychology, 25(5), 622-645.

Rodriguez-Fornells, A., Van Der Lugt, A., Rotte, M., Britti, B., Heinze, H.-J., \& Münte, T. F. (2005). Second language interferes with word production in fluent bilinguals: brain potential and functional imaging evidence. Journal of Cognitive Neuroscience, 17(3), 422-433.

Stasenko, A., Matt, G. E., \& Gollan, T. H. (2017). A relative bilingual advantage in switching with preparation: Nuanced explorations of the proposed association between bilingualism and task switching. Journal of Experimental Psychology: General, 146(11), 1527-1550.

Verhoef, K., Roelofs, A., \& Chwilla, D. J. (2009). Role of inhibition in language switching: Evidence from eventrelated brain potentials in overt picture naming. Cognition, 110(1), 84-99.

Verhoef, K. M., Roelofs, A., \& Chwilla, D. J. (2010). Electrophysiological evidence for endogenous control of attention in switching between languages in overt picture naming. Journal of Cognitive Neuroscience, 22(8), 18321843.

Wang, R., Fan, X., Liu, C., \& Cai, Z. G. (2016). Cognitive control and word recognition speed influence the Stroop effect in bilinguals. International Journal of Psychology, 51(2), 93101.

Wang, Y., Xue, G., Chen, C., Xue, F., \& Dong, Q. (2007). Neural bases ofasymmetric language switching in secondlanguage learners: An ER-fMRI study. NeuroImage, 35(2), $862-870$.

Ye, Y., Mo, L. E. I., \& Wu, Q. (2016). Mixed cultural context brings out bilingual advantage on executive function. Bilingualism: Language and Cognition, 20(4), 844-852.

Zhang, Y., Huang, P., Song, Z., Fang, L., Shen, T., Li, Y., Gong, Q., \& Xie, P. (2014). In-context language control with production tasks in bilinguals: an fMRI study. Brain Research, 1585, 131-140. 